\title{
Comparison of mucosal lining fluid sampling methods and influenza-specific IgA detection assays for use in human studies of influenza immunity
}

Thushan I. de Silva ${ }^{b, c, d}$, Victoria Gould ${ }^{a}$, Nuredin I. Mohammed ${ }^{d}$, Alethea Cope ${ }^{a}$, Adam Meijer ${ }^{\mathrm{e}}$, Ilse Zutt $^{\mathrm{e}}$, Johan Reimerink ${ }^{\mathrm{e}}$, Beate Kampmann ${ }^{\mathrm{b}, \mathrm{d}}$, Katja Hoschler ${ }^{\mathrm{f}}$, Maria Zambon $^{\mathrm{f}}$ \& John S. Tregoning ${ }^{a}$

a. Mucosal infection and Immunity, Section of Virology, Imperial College London, St Mary's Campus, London, W2 1PG, UK.

b. Section of Paediatrics, Imperial College London, St Mary's Campus, London, W2 1PG, UK.

c. Sheffield Teaching Hospitals NHS Foundation Trust, Royal Hallamshire Hospital, Glossop Road, Sheffield S10 2JF, UK.

d. Vaccines and Immunity Theme, Medical Research Council Unit The Gambia, PO Box 273, Banjul, The Gambia

e. Centre for Infectious Disease Research, Diagnostics and Screening (IDS)/PB22, National Institute for Public Health and the Environment (RIVM), Antonie van Leeuwenhoeklaan 9, 3721 MA Bilthoven, The Netherlands.

f. Virus Reference Department, Reference Microbiology Services, Public Health England, 61 Colindale Avenue, London NW9 5HT, UK.

Corresponding author:

T.I. de Silva

Section of Pediatrics, Imperial College London, St Mary's Campus, London, W2 1PG, UK

Email: t.de-silva@imperial.ac.uk

Keywords: IgA, ELISA, nasal 


\begin{abstract}
We need greater understanding of the mechanisms underlying protection against influenza virus to develop more effective vaccines. To do this, we need better, more reproducible methods of sampling the nasal mucosa. The aim of the current study was to compare levels of influenza virus A subtype-specific IgA collected using three different methods of nasal sampling. Samples were collected from healthy adult volunteers before and after LAIV immunization by nasal wash, flocked swabs and Synthetic Absorptive Matrix (SAM) strips. Influenza A virus subtype-specific IgA levels were measured by haemagglutinin binding ELISA or haemagglutinin binding microarray and the functional response was assessed by microneutralization. Nasosorption using SAM strips lead to the recovery of a more concentrated sample of material, with a significantly higher level of total and influenza H1-specific IgA. However, an equivalent percentage of specific IgA was observed with all sampling methods when normalised to the total IgA. Responses measured using a recently developed antibody microarray platform, which allows evaluation of binding to multiple influenza strains simultaneously with small sample volumes, were compared to ELISA. There was a good correlation between ELISA and microarray values. Material recovered from SAM strips was weakly neutralizing when used in an in vitro assay, with a modest correlation between the level of IgA measured by ELISA and neutralization, but a greater correlation between microarray-measured IgA and neutralizing activity. In conclusion we have tested three different methods of nasal sampling and show that flocked swabs and novel SAM strips are appropriate alternatives to traditional nasal washes for assessment of mucosal influenza humoral immunity.
\end{abstract}




\section{Introduction}

Whether acquired through natural infection or vaccination with live attenuated influenza vaccine (LAIV), antibody-mediated protection at mucosal surfaces is thought to play a key role in preventing influenza infections ${ }^{1}$. In particular IgA at mucosal surfaces has been shown to play a role in reducing viral shedding ${ }^{2}$. To support the assessment of mucosal influenza vaccines in future clinical trials and studies of naturally-acquired mucosal immunity, standardized sample collection methods from nasal mucosal lining fluid (MLF) are required that acceptable to participants (especially young children) and collect consistent MLF volumes. Furthermore, the volume collected and concentration of influenza-specific antibodies should be sufficient to measure mucosal responses to all influenza strains included in the vaccine. These aspects are also dependent on the requirements, performance and sensitivity of the IgA assay used, which traditionally has been based on ELISA techniques.

We present the results of a pilot study assessing different methods for collecting nasal MLF to detect both total and influenza-specific IgA: (i) nasal wash, the method traditionally used for this purpose but challenging in young children and subject to erratic sample recovery (ii) flocked swabs and (iii) synthetic absorptive matrix (SAM) strips. We also compare a highly sensitive ELISA modified from previous studies ${ }^{2,3}$ and a novel protein microarray requiring minimal sample volume, adapted to measure influenza-specific $\lg A^{4}$.

\section{Materials and Methods}

\subsection{Subjects, vaccination and mucosal lining fluid sampling methods}

Thirteen healthy adult volunteers (age 25 - 44 years, 8 male, 5 female) were recruited following written informed consent at the Clinical Research Facility, Sheffield Teaching Hospitals NHS Foundation Trust, Sheffield, UK. Ethical approval was granted by the Leeds East National Research Ethics Service Committee. Nasal MLF was sampled immediately prior to vaccination with 2015/16 seasonal tetravalent LAIV (Fluenz Tetra, Astra Zeneca, UK) and 28 days later via three methods: (i) Flocked swabs (Copan Diagnostics Inc., Murrieta, CA, USA) (ii) SAM strips (Hunt Diagnostics, UK) and (iii) Nasal washes using the Naclerio method ${ }^{5}$. A day 28 post-vaccine timepoint was chosen due to prior studies showing a mucosal IgA response to LAIV in both children and adults at approximately 1 month after vaccination ${ }^{6,7}$. Subjects were administered tetravalent LAIV into the nasal cavity $(0.1 \mathrm{ml}$ per nostril). This vaccine contained the haemagglutinin (HA) and 
neuraminidase (NA) from a A/California/7/2009 (H1N1) pdm09-like strain (A/Bolivia/559/2013) and $A /$ Switzerland/9715293/2013 $(H 3 N 2)$ as the two influenza $A$ antigens on the $A / A n n$ Arbor/6/60 attenuated backbone.

\subsection{Sample collection and processing}

All samples were collected by one operator, processed within 3 hours of collection and stored at $70^{\circ} \mathrm{C}$. The order of sample collection (nasal swab, strip, wash) was kept consistent at all visits with approximately 15 minute intervals in between. Sample processing was undertaken according to already established protocols using these methods. Nasal swabs were inserted into the nasal cavity and repeatedly rotated $360^{\circ}$ for approximately 5 seconds. Secretions were eluted by centrifugation at $4^{\circ} \mathrm{C}$ through a $\operatorname{Costar}^{\circledast}$ spin- $X^{\circledR}$ tube $(0.22 \mu \mathrm{m}$ pore size, Sigma, UK) pre-incubated with $300 \mu$ l of extraction buffer (EB: phosphate buffered saline (PBS) containing $0.02 \%$ sodium azide and $0.25 \mathrm{M} \mathrm{NaCl}$ ). Repeat centrifugation with a further $300 \mu \mathrm{l}$ of EB was performed. One swab from each nostril was obtained and eluted material pooled. A single SAM strip was inserted into a patent nostril according to the manufacturer's instructions for 60 seconds. Material was eluted in $300 \mu$ l of EB by centrifugation as detailed above. Material from one nostril at day 0 and both nostrils (processed separately) at day 28 were obtained. Nasal washes were obtained using the Naclerio method ${ }^{5}$, by instilling $10 \mathrm{ml}$ of sterile $0.9 \% \mathrm{NaCl}$ into each nostril in turn and asking the subject to expel forcefully into a sterile container. Collected material was centrifuged at $4^{\circ} \mathrm{C}$ and supernatant stored at $-70^{\circ} \mathrm{C}$.

\subsection{Enzyme-linked Immunosorbent Assays (ELISA)}

\subsubsection{Total IgA ELISA}

An ELISA was modified from previously described methods used to detect anti-HIV-1 antibodies

${ }^{8,9}$. ELISA plates (medium binding, Greiner Bio-One) were coated 1:1 with anti-Human kappa and anti-Human lambda capture antibodies (Southern Biotech, UK) at $4^{\circ} \mathrm{C}$ for $16-18$ hours. After washing in PBS-T (PBS with 0.05\% Tween ${ }^{\circledR} 20$ (Sigma, UK)), plates were blocked with assay buffer (AB; 1:1 Casein blocking buffer (Thermofisher scientific, UK) and PBS) for 1 hour at $37^{\circ} \mathrm{C} . \mathrm{MLF}$ samples were diluted 1:1,000, 1:5,000 and 1:25,000 and incubated for 1 hour at $37^{\circ} \mathrm{C}$ in triplicate, along with a serial dilution of human IgA (from colostrum, Sigma, UK) to generate a standard curve (range $200 \mathrm{ng} / \mathrm{ml}$ to $0.195 \mathrm{ng} / \mathrm{ml}$ ). After washing with PBS-T, IgA antibodies were detected using biotin-labelled Goat anti-human IgA (Insight Biotechnology Ltd, UK) followed by 
streptavidin-poly-HRP40 (2BScientific, UK) and signal developed using SureBlue TMB Microwell Peroxidase Substrate and TMB Stop solution (both from Insight Biotechnology Ltd, UK). After development, plates were read at $450 \mathrm{~nm}$ using a VersaMax plate reader. Generation of the standard curve and total IgA quantification in samples was performed following subtraction of background optical density (OD) values. A mean estimated IgA value of dilutions falling within the linear portion of the standard curve were included in final calculations. Some SAM stripcollected material required further dilution to $1: 125,000$ to obtain OD values within the linear portion of the standard curve.

\subsubsection{Influenza-specific IgA ELISA}

ELISA plates were coated with recombinant HA from either A/California/7/2009 (full length HA) or A/Switzerland/9715293/2013 H3N2 (HA1) at $2.0 \mu \mathrm{g} / \mathrm{ml}$ (Sino Biological, China) at $4^{\circ} \mathrm{C}$ for $16-$ 18 hours. After washing and blocking steps as above, MLF samples were incubated neat, at 1:5, 1:25 or 1:125 dilution in triplicate for 1 hour at $37^{\circ} \mathrm{C}$. IgA detection, development and quantification using an IgA standard curve was performed as described above.

\subsection{Haemagglutinin Microarray to detect anti-influenza IgA antibodies}

A previously described HA microarray designed to profile anti-influenza IgG antibodies in human sera $^{4}$ was adapted to detect anti-influenza IgA in MLF samples. Briefly, nitrocellulose coated slides (ONCYTE ${ }^{\circledR}$ AVID, Grace Bio-Labs, USA) were spotted in duplicate with recombinant HA1 protein from both A/California/6/2009 (H1N1pdm09) and A/Brisbane/10/2007 (H3N2). These were used to detect anti-influenza IgA in 40 operator-blinded samples (consisting of day 0 and day 28 swab and strip-eluted material from 10 subjects). Slides were treated with Blocker ${ }^{\mathrm{TM}}$ BLOTTO blocking buffer (Thermo Fisher Scientific, USA) for 1 hour at $37^{\circ} \mathrm{C}$. After washing, samples were diluted 1:3 and transferred to the arrays. Following incubation for 1 hour at $37^{\circ} \mathrm{C}$, slides were washed and incubated with goat anti-human IgA conjugated with Dylight649-fluorescent dye (Jackson ImmunoResearch, USA) for a further 1 hour at $37^{\circ} \mathrm{C}$. After washing, slides were dried and signals quantified by a PowerScanner ${ }^{\mathrm{TM}}$ microarray scanner (Tecan Trading AG, Switzerland). Mean spot fluorescence foreground intensity was determined by using ScanArray ${ }^{\circledR}$ Express software (PerkinElmer, USA).

\subsection{Influenza microneutralization assays}


MLF from SAM strip-eluted material was tested by standard methods using a microneutralization (MN) assay as described previously ${ }^{10}$, with egg-cultured A/California/7/2009 H1N1pdm09 virus. Briefly, samples were heat inactivated for $30 \mathrm{~min}$ at $56^{\circ} \mathrm{C}$ and 2-fold serial dilutions from 1:10 to 1:320 in $50 \mu \mathrm{l}$ volume of PBS (in duplicate) were mixed with an equal volume of influenza virus previously titrated on Madin-Darby canine kidney (MDCK) cells. After a 1-hour incubation at room temperature, $100 \mu \mathrm{l}$ of MDCK cells at $5 \times 10^{5} / \mathrm{ml}$ was added to each well and plates incubated for $16-18$ hours at $37{ }^{\circ} \mathrm{C}$. Monolayers were washed and fixed in cold methanol/ $\mathrm{H}_{2} \mathrm{O}_{2}(0.6 \%)$ for 30 minutes. The presence of influenza nucleoprotein (NP) was detected by ELISA with a monoclonal antibody to the influenza A NP as described previously ${ }^{11,12}$. MN titres were calculated as the MLF dilution at which $50 \%$ reduction in the virus-infected to uninfected optical density (OD) ratio was seen.

\subsection{Statistical analyses}

Based on a previous study comparing allergen-specific IgA detection in SAM strips and nasal washes $^{13}$, we calculated that 5 participants would be sufficient to detect a 2 -fold difference in anti-influenza IgA levels at day 28 post-LAIV between these two methods (power $90 \%$, alpha 0.01). To account for a proportion of subjects without detectable IgA and potential dropouts, we aimed to recruit 10 - 15 participants. All statistical analyses were performed using GraphPad Prism version 5.0 for Macintosh. Differences in IgA levels among the three sampling methods were investigated using the Friedman's test. The non-parametric pairwise multiple comparisons procedure, Dunn's test, was used for post-hoc comparison whenever the null hypothesis was rejected based on Friedman's test. Correlation analyses were made using the Spearman's rank correlation coefficient.

\section{Results}

\subsection{The yield of total and H1pdm09-specific IgA is greater in SAM strips compared to flocked swabs or nasal washes}

Approximately $2-18 \mathrm{ml}$ from nasal washes, $1.2 \mathrm{ml}$ from flocked swabs and $300 \mu \mathrm{l}$ from SAM strip collected fluid was available for assays. Considering both day 0 and day 28 values $(n=26)$, total IgA concentration was 4-6 fold higher in SAM strip-collected material compared to flocked swab or nasal wash material: median (inter-quartile range, IQR) $348.0 \mu \mathrm{g} / \mathrm{ml}(171.4,509.3), 78.3 \mu \mathrm{g} / \mathrm{ml}$ 
$(32.3,137.0)$ and $55.9 \mu \mathrm{g} / \mathrm{ml}(24.5,90.4)$ respectively. H1pdm09 HA-specific IgA concentration was approximately 5-fold higher in SAM strip-collected material: median $118.1 \mathrm{ng} / \mathrm{ml}$ (IQR 40.9, 259.7), $23.4 \mathrm{ng} / \mathrm{ml}(7.1,64.9)$ and $25.4 \mathrm{ng} / \mathrm{ml}(11.1,35.4)$ in SAM strip, swab and wash samples respectively. Significant differences between sampling methods are shown in Figure 1a and 1b. Total IgA values for each subject were more consistent across visits in flocked swab and SAM strip samples than nasal wash samples, with a significant correlation seen between day 0 and day 28 total IgA quantities for each subject in samples collected by swab and strip (Figure $1 c-e)$. Finally, total IgA quantities in samples collected from left and right nares using SAM strips at day 28 were compared and no significant difference found (median 335.0 vs $228.7 \mu \mathrm{g} / \mathrm{ml}, \mathrm{p}=0.45$ ), with a good correlation between the two $\left(r_{s} 0.736, p=0.002\right)$ (data not shown).

\subsection{Standardization of H1pdm09-specific IgA to total IgA results in comparable values between sampling methods}

To account for variability in the volume of MLF collected across subjects and visits, H1pdm09 HAspecific IgA quantities were expressed as a percentage of total IgA. These were more comparable between nasal swabs and SAM strips than raw values at both day 0 and day 28 (Fig 2). The percentage H1pdm09 HA-specific IgA in nasal washes was, however, significantly higher than in flocked swabs at day 28 . This may reflect the trend towards a greater yield of total IgA in swabs compared to washes (Fig 1a). A good correlation is seen between percentage H1pdm09 HAspecific/total IgA values between methods in each subject (Fig 3), with the most comparable being flocked swab and SAM strips, again suggesting that these are more comparable and consistent methods of MLF collection.

\subsection{Comparison of haemagglutinin microarray and ELISA to detect anti-influenza IgA and the mucosal response to LAIV}

A disadvantage of ELISA is the sample volume required per antigen tested, making it challenging to measure antibodies to all antigens included in seasonal influenza vaccines. HA microarray ${ }^{4}$ is able to reliably detect anti-influenza IgG in very small quantities of serum $(10 \mu l)$, so we tested the ability of this assay to detect anti-influenza IgA in MLF. Due to sample volume availability, H3 HAspecific IgA ELISAs were only performed on flocked swab (and not SAM strip) material. A good correlation was seen between both H1pdm09 HA and H3 HA influenza-specific IgA levels 
(fluorescence units) in the HA microarray and IgA quantified by ELISA in $\mathrm{ng} / \mathrm{ml}$ (Fig 4). This was in spite of a mismatch in H3 HA antigen in the microarray slides prepared for a previous study (A/Brisbane/10/2007) and that of the vaccine antigen (A/Switzerland/9715293/2013).

To assess the mucosal IgA response to LAIV, the fold difference was calculated between day 28 and day 0 samples in influenza-specific IgA measured by ELISA and HA microarray for each subject (in each case normalized by the total IgA quantity). A $\geq 2$-fold increase was considered a significant response as defined in a previous LAIV study ${ }^{6}$. This was undertaken for swab samples in the 10 subjects for whom comparable H1pdm09 and H3-specific data were available (Supplementary Table 1). No subject showed a significant increase in H1pdm09 HA-specific IgA post-vaccine using ELISA-measured data, with one subject showing an H3-specific IgA response. In contrast, three subjects showed an $\mathrm{H} 1$-specific IgA response and three subjects an $\mathrm{H} 3-$ specific IgA response to LAIV using HA microarray measured data (with two subjects showing a response to both antigens).

\subsection{Low titre influenza microneutralization activity is detectable in nasal mucosal lining fluid}

To evaluate a more functional readout of anti-influenza activity in MLF, available SAM stripcollected material (as those with the greatest antibody yield) were tested in a standard influenza microneutralization (MN) assay. While $11 / 25$ of samples tested showed detectable titres against A/California/7/2009 H1N1pdm09 virus, these were all low titre (1:10 - 1:24). A weak but significant correlation was observed between the reciprocal MN titre and H1pdm09 HA-specific IgA value quantified by ELISA (Fig 5a). Interestingly, a greater correlation was observed between reciprocal MN titres and $\mathrm{H} 1 \mathrm{pdm} 09 \mathrm{HA}$-specific IgA levels (fluorescence units) detected in the HA microarray (Fig 5b).

\section{Discussion}

Reliable, high-yield, participant-acceptable methods to assess mucosal anti-influenza humoral activity are vital in studies of both natural infection and vaccine-induced immunity. We provide (to our knowledge for the first time) a direct comparison of nasal washes with flocked swabs and SAM strips to collect MLF and measure influenza-specific IgA responses, with the latter two 
representing techniques with greater convenience for both the investigator and study participant.

We demonstrate that the yield of total and influenza-specific IgA is significantly higher in SAM strips compared to flocked swabs or nasal washes. This is in keeping with previous reports of greater cytokine ${ }^{13,14}$ and allergen-specific antibody ${ }^{13}$ recovery using SAM strips, but in contrast to a recent study showing SAM strips had lower HIV-1 antibody recovery from nasal turbinates compared to flocked swabs ${ }^{8}$. The reasons for this difference are not clear, but could include how long SAM strips remain in contact with the nasal mucosa, environmental variation affecting mucosal moistness and pre-incubation of $\operatorname{Costar}^{\circledR}$ spin- $X^{\circledR}$ tubes with elution buffer, all of which may affect antibody yield. Despite the known variability of nasal IgA secretion (including diurnally ${ }^{15}$ ), we also observed a significant correlation between day 0 and day 28 total $\operatorname{lgA}$ recovery for each subject in flocked swab and SAM strip samples, but not nasal washes. This supports the hypothesis that swabs and SAM strips are more consistent sample collection methods compared to washes.

Given the small volumes of nasal MLF obtainable, sensitive assays are required to measure influenza-specific humoral immunity in these samples. One potential drawback of ELISA is the high volume of sample required, especially if low antibody levels necessitate running undiluted material and IgA specific to several antigens are measured. We therefore evaluated the ability of a protein microarray ${ }^{4}$ to detect nasal influenza-specific $\operatorname{lgA}$ and demonstrated an excellent correlation with ELISA data. Furthermore, this assay was able to quantify IgA (in fluorescence units) in samples with a wide range of ELISA-quantified IgA values $(2.7 \mathrm{ng} / \mathrm{ml}-1437 \mathrm{ng} / \mathrm{ml})$ at a single sample dilution (1:3), thus reducing the potential errors introduced by requiring multiple different dilutions across samples to fall within a range detectable by ELISA. This methodology warrants further assessment in larger studies and has the potential to simultaneously measure not only anti-HA IgA against several influenza type A subtypes and influenza B lineages, but also against other antigenic targets such as neuraminidase.

We also sought to measure functional responses in SAM strip samples by using a standard influenza MN assay. Although neutralizing antibody was detected in $44 \%$ of samples tested, titres were low, suggesting that this is not a suitable method to assess functional responses in nasal 
MLF despite the higher antibody yield in SAM strip samples. We found only a weak correlation between ELISA-quantified influenza-specific IgA and MN titres. While this could be due to the lack of precision in $\mathrm{MN}$ titres at such a restricted range, it could also be explained by varying degrees of functional activity in binding IgA antibodies, which may limit the spread of budding virus from the cell, rather than classical entry inhibiting neutralization ${ }^{16}$. IgG, unmeasured in this study, may make a more significant contribution to neutralization measured by the in vitro assay used. Interestingly, the correlation between MN activity and $\mathrm{H} 1 \mathrm{pdm} 09$ influenza-specific IgA detected in the microarray was greater. Whether microarray-detected IgA levels are a better surrogate marker of neutralizing activity in nasal MLF than ELISA-quantified values requires further investigation. There remains a need to combine a functional assay with a convenient MLF sampling method.

Finally, although the main purpose of the study was to evaluate sampling methods and antiinfluenza IgA detection assays, we assessed the mucosal IgA response to the H1N1pdm09 and H3N2 components in tetravalent LAIV in the limited sample of adults in the study. Using a $\geq 2$-fold increase from baseline post-LAIV as a significant response ${ }^{6}$, none of ten subjects showed a H1N1pdm09-specific response, with one subject showing a H3N2-specific response in ELISAmeasured influenza-specific IgA data. This is consistent with previous reports of lower LAIV efficacy in adults compared to children ${ }^{17}$ and poor antibody responses to H1N1pdm09 compared to $\mathrm{H} 3 \mathrm{~N} 2$ or influenza $\mathrm{B}$ components in LAIV even in children ${ }^{18,19}$. Interestingly, when we considered the influenza-specific IgA data generated by HA microarray, three subjects showed a significant response to $\mathrm{H} 1 \mathrm{HA}$ and three subjects to H3 HA (2 subjects showed a $\geq 2$-fold increase to both $\mathrm{H} 1 \mathrm{pdm} 09$ and $\mathrm{H} 3 \mathrm{HA}$ ). It is difficult to conclude from these data whether the ELISA or microarray data reflect a more biologically relevant result and further studies are required to validate the HA microarray assay in particular.

Given the recent varying effectiveness ${ }^{20-22}$ and efficacy ${ }^{23,24}$ of both the Ann Arbor and Leningrad backbone LAIV preparations, there is an urgent need to conduct studies which incorporate reliable mucosal immunogenicity endpoints following LAIV. Studies of natural influenza infection, as well as human influenza challenge models can also enhance our knowledge of anti-influenza mucosal immunity. We provide novel data on both sampling methods and IgA assays for use in such studies. 

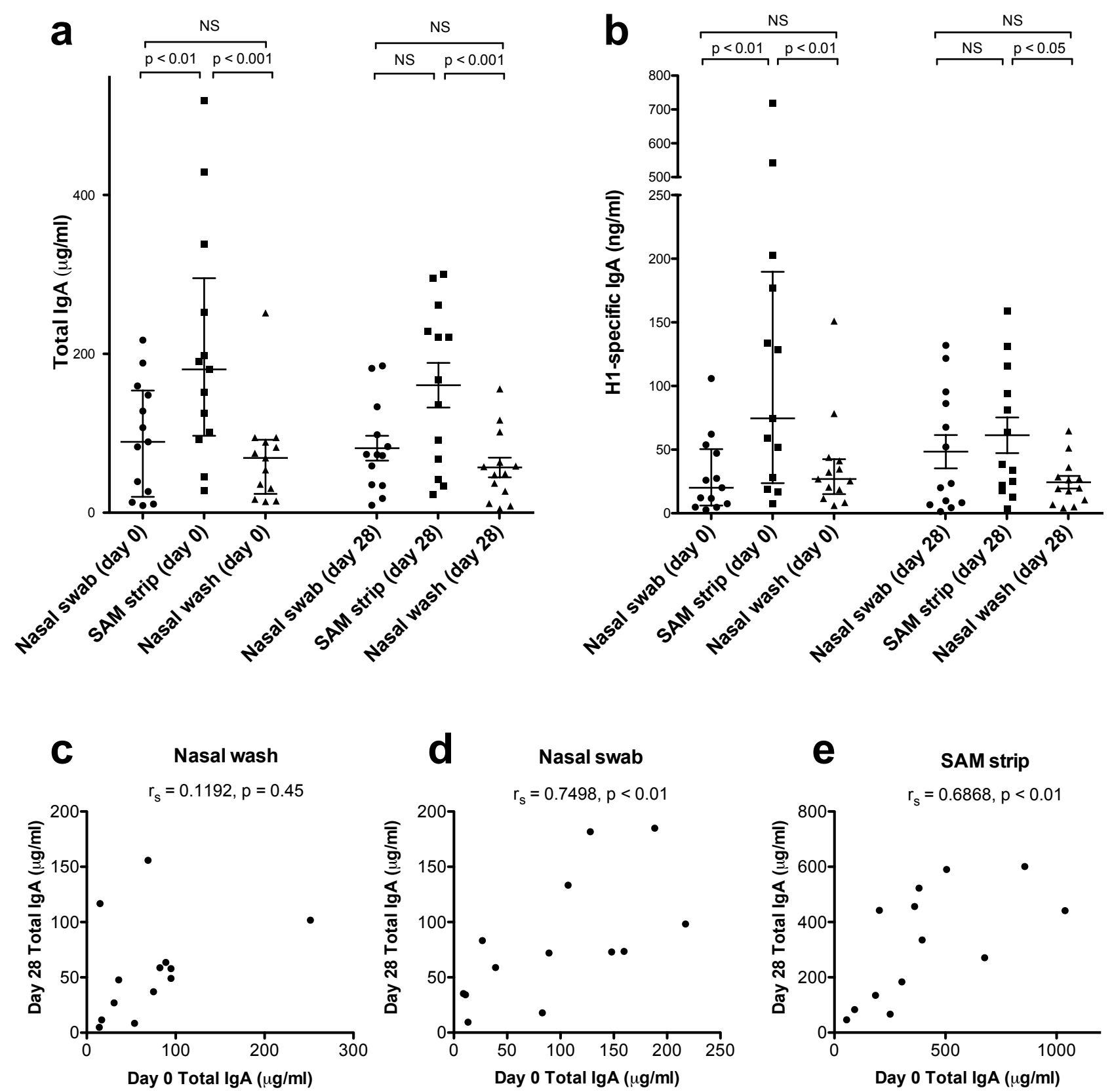

Figure 1. SAM strips recover more concentrated levels of influenza specific IgA. Total IgA (a) and $\mathrm{H} 1$-specific IgA (b) in nasal swabs, SAM strips and nasal washes quantified by indirect ELISA (Friedman test with Dunn's posttest for multiple comparisons) at day 0 and day 28. Comparisons include samples from 13 subjects. Values shown for SAM strip yield are corrected so they are comparable with flocked swab yield, given differences in elution volume of the two methods (i.e. SAM strip values halved as each SAM strip was eluted in $300 \mu l$ while each nasal swab was eluted in $600 \mu \mathrm{l}$ ). Median and inter-quartile range are shown for each dataset. Correlation between the total IgA yield at day 0 and day 28 from from each subject $(n=13)$ in nasal wash (c), swab (d) and SAM strip (e) samples is shown. SAM = synthetic absorptive material. NS = not significant. 


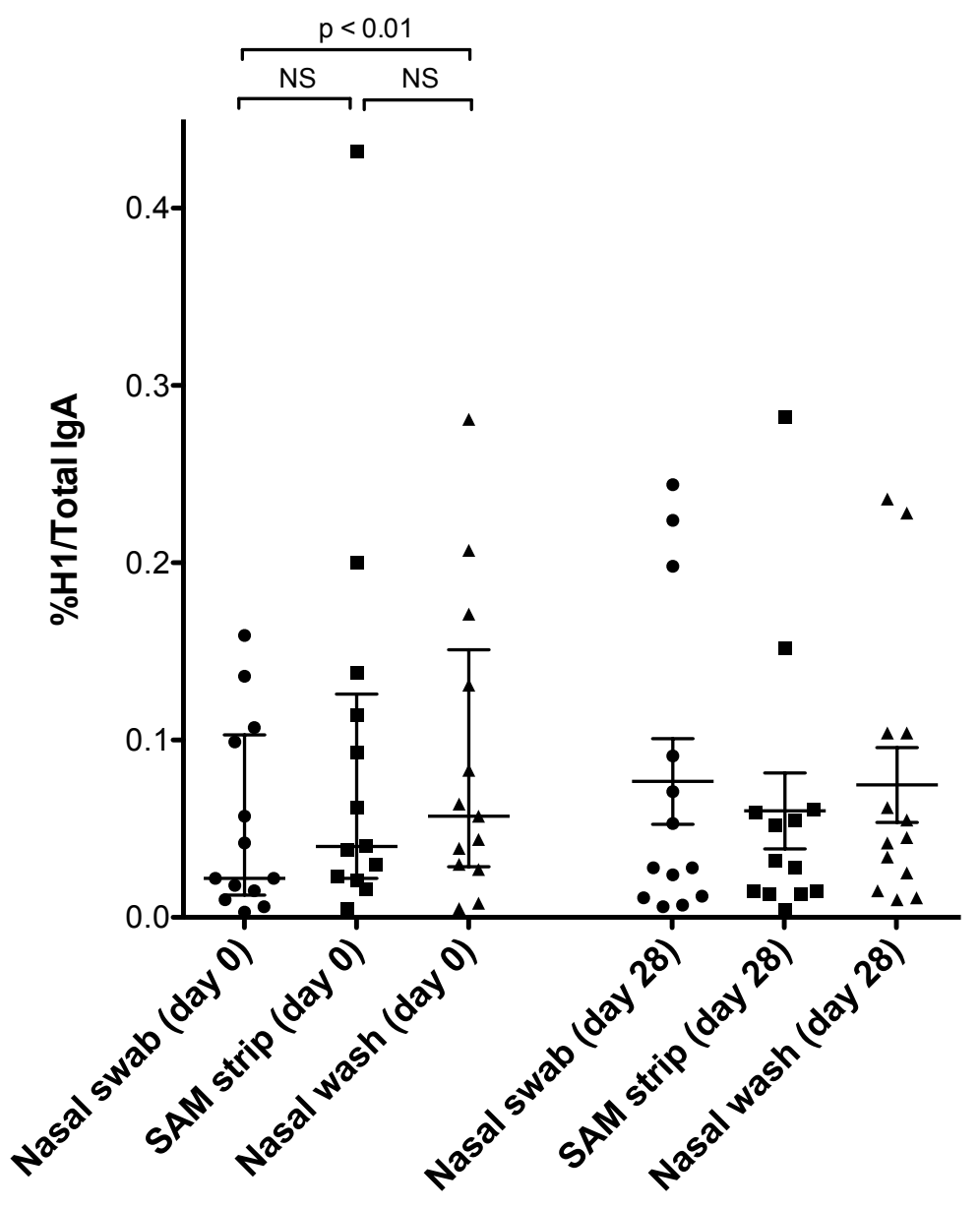

Figure 2. The proportion of specific IgA as a percentage of total IgA is constant regardless of sampling method. Comparison of total H1-specific IgA yield from nasal swabs, SAM strips and nasal washes, expressed as a \% of total IgA detected in the same sample at day 0 and 28 . Values were quantified by indirect ELISA and comparisons made using the Friedman test with Dunn's posttest for multiple comparisons. Overall Friedman statistic for day 28 samples was not significant, therefore post-test multiple comparisons were not performed. Comparisons include samples from 13 subjects. Median and inter-quartile range are shown for each dataset. NS = not significant. SAM = synthetic absorptive material. 
a Nasal swab vs nasal wash (day 0)

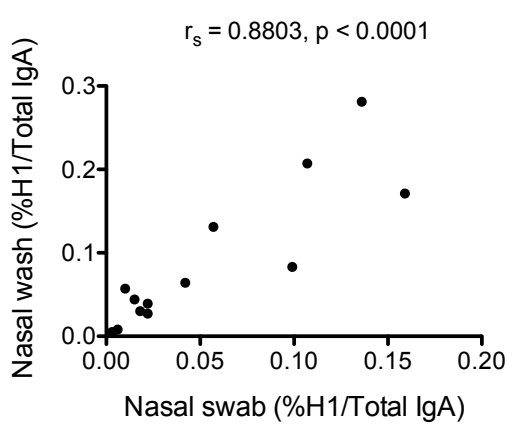

d Nasal swab vs nasal wash (day 28)

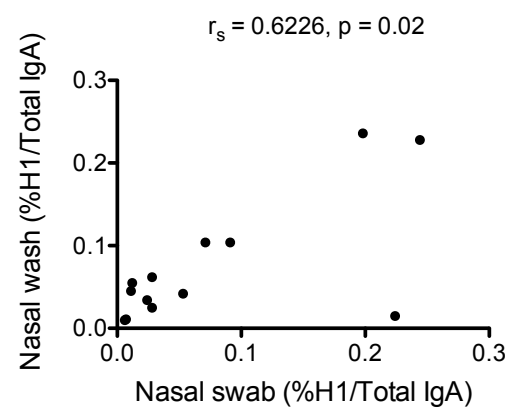

b SAM strip vs nasal wash (day 0 )

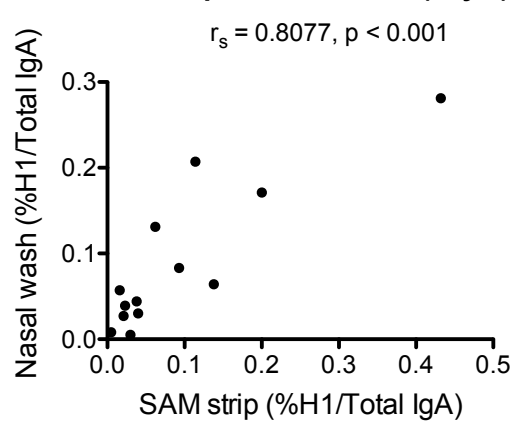

e SAM strip vs nasal wash (day 28)

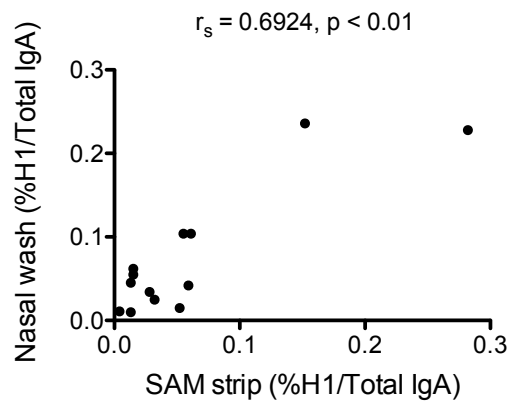

C SAM strip vs nasal swab (day 0)

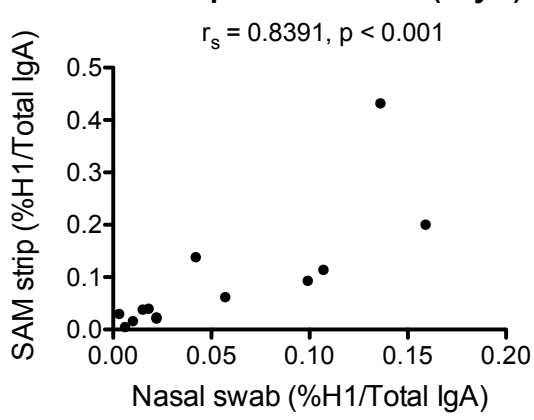

f

SAM strip vs nasal swab (day 28)

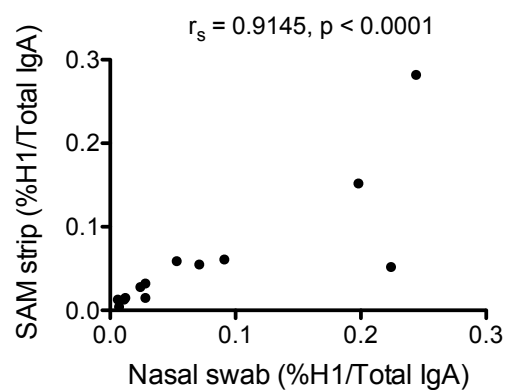

Figure 3. Correlation between the H1-specific IgA yield (expressed as a \% of total IgA) between different sampling methods at day 0 ( $a, b$ and $c$ ) and day 28 ( $d$, e and f). Comparisons include samples from 13 subjects. SAM = synthetic absorptive material. 

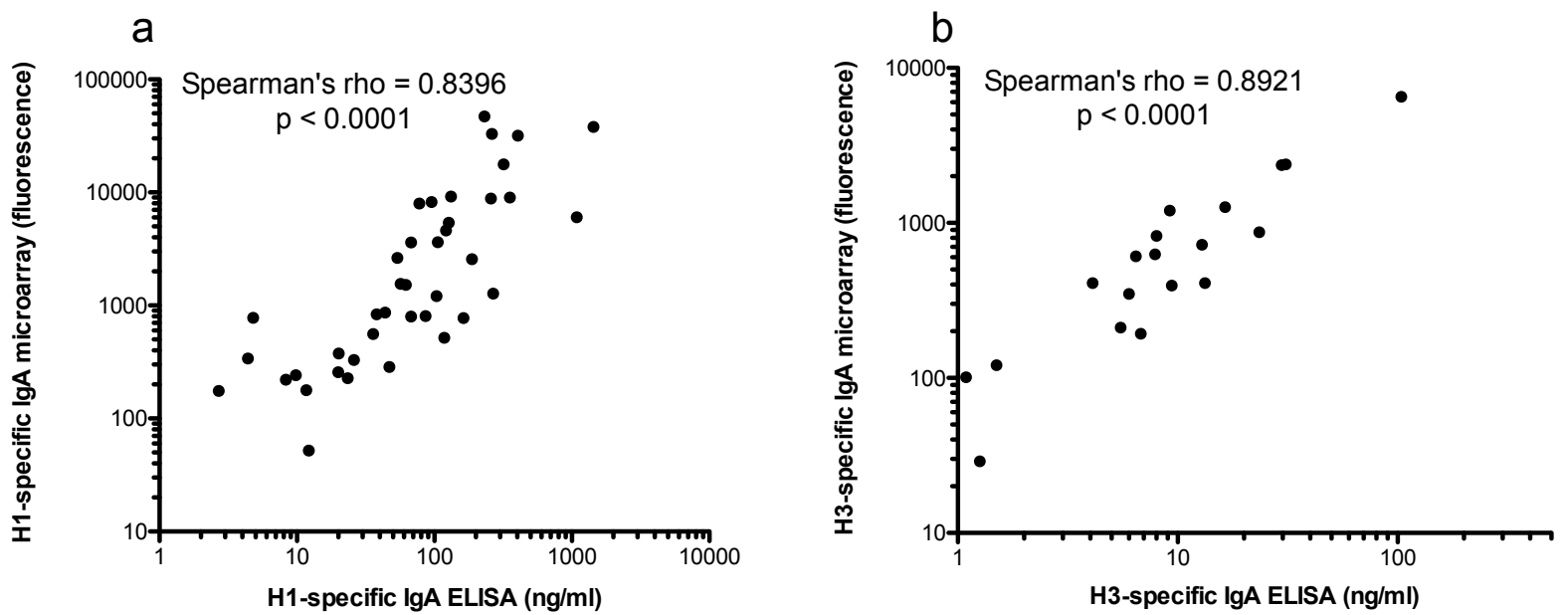

Figure 4. Influenza specific IgA levels detected are similar when measured by ELISA or microarray. Correlations between H1-specific (a) and H3-specific (b) IgA in mucosal lining fluid samples detected by protein microarray and ELISA. H1-specific comparisons include pre and postvaccine SAM strip and flocked swab samples from 10 subjects $(n=40)$. H3-specific comparisons include pre and post-vaccine flocked swab samples from 10 subjects $(n=20)$.

a

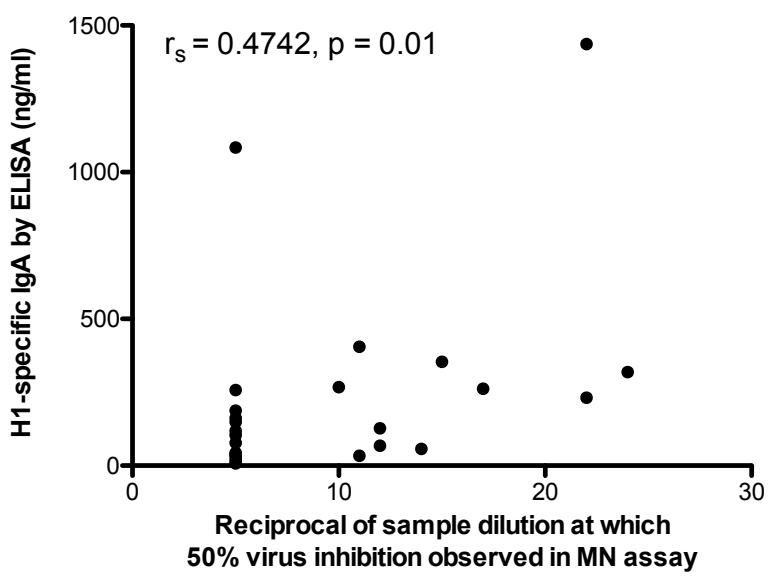

b

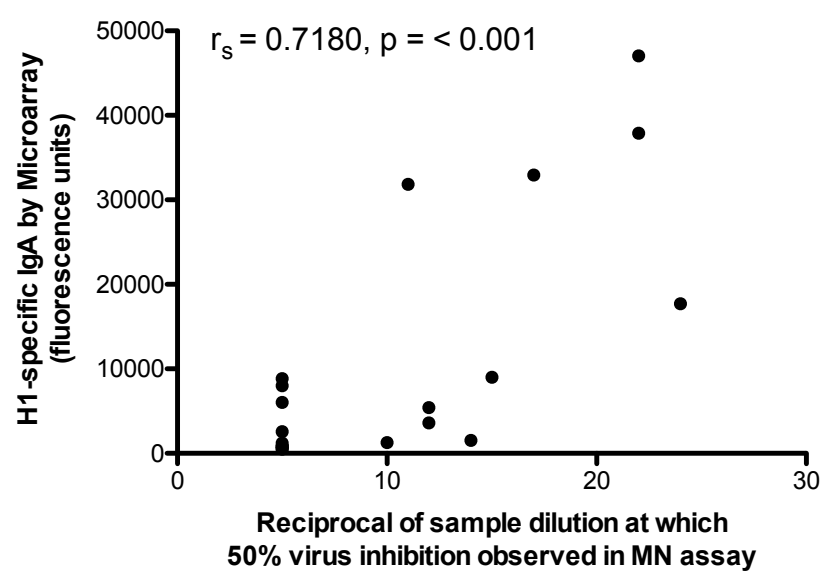

Figure 5. IgA recovered from Nasal samples correlates with neutralization. Correlation between $\mathrm{H} 1$-specific IgA yield in SAM strips quantified by ELISA (a) or haemagglutinin microarray (b) and neutralizing activity against A/California/7/2009 H1N1 virus as assessed in a microneutralization (MN) assay (a). Samples with titres $<10$ are assigned a titre of 1:5 for the purpose of the analysis above. 
Supplementary Table 1. The H1- and H3-specific IgA response to LAIV measured by ELISA and protein microarray. Response is expressed as fold-change from day 0 (baseline) to day 28 after normalization by total IgA quantity in the sample. Shown are data generated from flocked swabs. Responses with $\geq 2$-fold increase in normalized IgA following LAIV are highlighted.

\begin{tabular}{|c|c|c|c|c|c|c|}
\hline \multirow[t]{2}{*}{ Subject } & \multicolumn{2}{|c|}{$\begin{array}{c}\text { Normalized influenza-specific } \\
\text { IgA fold-change measured by } \\
\text { ELISA (day 28/day 0) }\end{array}$} & \multicolumn{2}{|c|}{$\begin{array}{c}\text { Normalized influenza-specific } \lg A \\
\text { fold-change measured by } \\
\text { microarray (day 28/day } 0)^{\#}\end{array}$} & \multicolumn{2}{|c|}{ Total IgA $(\mu \mathrm{g} / \mathrm{ml})$} \\
\hline & H1 & H3 & H1 & H3 & Day 0 & Day 28 \\
\hline 1 & 1.25 & 1.26 & 1.81 & 1.63 & 89 & 72 \\
\hline 2 & 1.80 & 1.21 & 3.94 & $+\dagger$ & 9 & 35 \\
\hline 3 & 1.99 & 1.72 & 0.95 & 0.80 & 160 & 74 \\
\hline 4 & 1.41 & 1.26 & 4.02 & 2.49 & 39 & 59 \\
\hline 5 & 1.10 & 1.17 & 1.76 & 1.17 & 217 & 98 \\
\hline 6 & 1.25 & 1.58 & 2.20 & 2.07 & 128 & 182 \\
\hline 7 & 0.65 & 0.84 & 1.36 & 1.04 & 148 & 73 \\
\hline 8 & 0.92 & 12.93 & 1.02 & 8.59 & 107 & 133 \\
\hline 9 & 1.16 & $\dagger$ & 0.44 & 0.28 & 27 & 83 \\
\hline 10 & 1.84 & 1.99 & 1.43 & 1.04 & 11 & 34 \\
\hline
\end{tabular}

\# (Specific lgA at day $28(\mathrm{ng} / \mu \mathrm{L}$ in ELISA or fluorescence units in microarray)/total lgA on day $28(\mu \mathrm{g} / \mathrm{ml})$

(Specific $\lg A$ at day 0 (ng/uL in ELISA or fluorescence units in microarray)/total IgA on day $0(\mu \mathrm{g} / \mathrm{ml})$

$\dagger$ Unable to measure fold-change as no detectable $\mathrm{H} 3$-specific IgA at baseline.

†† Unable to calculate fold-change as background corrected fluorescence value negative at baseline.

\section{Acknowledgements}

We thank all volunteers who took part in the study. We thank Dr. Trevor Hansel for advice on the use of SAM strips and Dr. Daniela Ferreira and Dr. Jenna Gritzfeld for advice on nasal wash technique and sample processing. This work was funded by the Academy of Medical Sciences via a starter grant awarded to Thushan de Silva and carried out at the National Institute for Health Research (NIHR) Sheffield Clinical Research Facility. The IgA ELISA assay was established with support from an NIHR BRC grant to Imperial College London. The views expressed are those of the authors and not necessarily those of the Academy of Medical Sciences, NHS, the NIHR or the Department of Health, UK. Thushan de Silva is currently funded by a Wellcome Trust Intermediate Clinical Fellowship (110058/Z/15/Z). 


\section{References}

1. Hasegawa $H$, van Reit E, Kida H. Mucosal immunization and adjuvants. Curr Top Microbiol Immunol 2015;386:371-80.

2. Gould VMW, Francis JN, Anderson KJ, Georges B, Cope AV, Tregoning JS. Nasal IgA Provides Protection against Human Influenza Challenge in Volunteers with Low Serum Influenza Antibody Titre. Front Microbiol 2017;8:900.

3. Donnelly L, Curran RM, Tregoning JS, et al. Intravaginal immunization using the recombinant HIV-1 clade-C trimeric envelope glycoprotein CN54gp140 formulated within lyophilized solid dosage forms. Vaccine 2011;29:4512-20.

4. Koopmans $M$, de Bruin E, Godeke GJ, et al. Profiling of humoral immune responses to influenza viruses by using protein microarray. Clinical microbiology and infection : the official publication of the European Society of Clinical Microbiology and Infectious Diseases 2012;18:797807.

5. Naclerio RM, Meier HL, Kagey-Sobotka A, et al. Mediator release after nasal airway challenge with allergen. Am Rev Respir Dis 1983;128:597-602.

6. Ambrose CS, Wu X, Jones T, Mallory RM. The role of nasal IgA in children vaccinated with live attenuated influenza vaccine. Vaccine 2012;30:6794-801.

7. Barria MI, Garrido JL, Stein C, et al. Localized mucosal response to intranasal live attenuated influenza vaccine in adults. J Infect Dis 2013;207:115-24.

8. Bergin PJ, Langat R, Omosa-Manyonyi G, et al. Assessment of Anti-HIV-1 Antibodies in Oral and Nasal Compartments of Volunteers From 3 Different Populations. J Acquir Immune Defic Syndr 2016;73:130-7.

9. Cranage MP, Fraser CA, Cope A, et al. Antibody responses after intravaginal immunisation with trimeric HIV-1 CN54 clade C gp140 in Carbopol gel are augmented by systemic priming or boosting with an adjuvanted formulation. Vaccine 2011;29:1421-30.

10. Rowe T, Abernathy RA, Hu-Primmer J, et al. Detection of antibody to avian influenza A (H5N1) virus in human serum by using a combination of serologic assays. J Clin Microbiol 1999;37:937-43.

11. Miller E, Hoschler K, Hardelid P, Stanford E, Andrews N, Zambon M. Incidence of 2009 pandemic influenza A H1N1 infection in England: a cross-sectional serological study. Lancet 2010;375:1100-8.

12. Waddington CS, Walker WT, Oeser C, et al. Safety and immunogenicity of ASO3B adjuvanted split virion versus non-adjuvanted whole virion $\mathrm{H} 1 \mathrm{~N} 1$ influenza vaccine in UK children aged 6 months-12 years: open label, randomised, parallel group, multicentre study. BMJ 2010;340:c2649.

13. Scadding GW, Calderon MA, Bellido V, et al. Optimisation of grass pollen nasal allergen challenge for assessment of clinical and immunological outcomes. J Immunol Methods 2012;384:25-32.

14. Jochems SP, Piddock K, Rylance J, et al. Novel Analysis of Immune Cells from Nasal Microbiopsy Demonstrates Reliable, Reproducible Data for Immune Populations, and Superior Cytokine Detection Compared to Nasal Wash. PLoS One 2017;12:e0169805.

15. Mygind N, Thomsen J. Diurnal variation of nasal protein concentration. Acta Otolaryngol 1976;82:219-21. 
16. Mazanec $\mathrm{MB}$, Coudret $\mathrm{CL}$, Fletcher DR. Intracellular neutralization of influenza virus by immunoglobulin A anti-hemagglutinin monoclonal antibodies. J Virol 1995;69:1339-43.

17. Osterholm MT, Kelley NS, Sommer A, Belongia EA. Efficacy and effectiveness of influenza vaccines: a systematic review and meta-analysis. Lancet Infect Dis 2012;12:36-44.

18. Mohn KG, Bredholt G, Brokstad KA, et al. Longevity of B-Cell and T-Cell Responses After Live Attenuated Influenza Vaccination in Children. J Infect Dis 2014.

19. Mohn KG, Brokstad KA, Pathirana RD, et al. Live Attenuated Influenza Vaccine in Children Induces B-Cell Responses in Tonsils. J Infect Dis 2016;214:722-31.

20. Nohynek H, Baum U, Syrjanen R, Ikonen N, Sundman J, Jokinen J. Effectiveness of the live attenuated and the inactivated influenza vaccine in two-year-olds - a nationwide cohort study Finland, influenza season 2015/16. Euro Surveill 2016;21.

21. Pebody R, Warburton F, Ellis J, et al. Effectiveness of seasonal influenza vaccine for adults and children in preventing laboratory-confirmed influenza in primary care in the United Kingdom: 2015/16 end-of-season results. Euro Surveill 2016;21.

22. Zimmerman RK, Nowalk MP, Chung J, et al. 2014-2015 Influenza Vaccine Effectiveness in the United States by Vaccine Type. Clin Infect Dis 2016.

23. Brooks WA, Zaman, K., Lewis, K.D.C., Ortiz, J.R., Goswami, D., Feser, J., Tahia Sharmeen, A., Nahar, K., Rahman, M., Rahman, M.Z., Barin, B., Yunus, M., Fry, A.M., Bresee, J., Azim, T., Neuzil, K.M. Efficacy of Russian-backbone live attenuated influenza vaccine among young children in Bangladesh: a randomised, double-blind, placebo-controlled trial. Lancet Global Health 2016. 24. Victor JC, Lewis, K.D.C., Diallo, A., Niang, M.N., Diarra, B., Dia, N., Ortiz, J.R., Widdowson, M., Feser, J., Hoagland, R., Emery, S.L., Lafond, K.E., Neuzil, K.M. Efficacy of Russian-backbone live attenuated influenza vaccine among children in Senegal: a randomised, double-blind, placebocontrolled trial. Lancet Global Health 2016. 